\title{
Transformation of cyclodextrin glucanotransferase (CGTase) from aqueous suspension to fine solid particles via electrospraying
}

\begin{abstract}
In this study, the potential of electrohydrodynamic atomization or electrospraying to produce nanometer-order CGTase particles from aqueous suspension was demonstrated. CGTase enzyme was prepared in acetate buffer solution $(1 \% \mathrm{v} / \mathrm{v})$, followed by electrospraying in stable Taylor cone-jet mode. The deposits were collected on aluminium foil (collector) at variable distances from the tip of spraying needle, ranging from 10 to $25 \mathrm{~cm}$. The Coulomb fission that occurs during electrospraying process successfully transformed the enzyme to the solid state without any functional group deterioration. The functional group verification was conducted by FTIR analysis. Comparison between the deposit and the as-received enzyme in dry state indicates almost identical spectra. By increasing the distance of the collector from the needle tip, the average particle size of the solidified enzyme was reduced from $200 \pm 117$ $\mathrm{nm}$ to $75 \pm 34 \mathrm{~nm}$. The average particle sizes produced from the droplet fission were in agreement with the scaling law models. Enzyme activity analysis showed that the enzyme retained its initial activity after the electrospraying process. The enzyme particles collected at the longest distance $(25 \mathrm{~cm})$ demonstrated the highest enzyme activity, which indicates that the activity was controlled by the enzyme particle size.
\end{abstract}

Keyword: Electrospraying; Droplet fission; Solidified enzyme; Enzyme activity 